Original Article

\title{
ZOLMITRIPTAN BRAIN TARGETING VIA INTRANASAL ROUTE USING SOLID LIPID NANOPARTICLES FOR MIGRAINE THERAPY: FORMULATION, CHARACTERIZATION, IN-VITRO AND IN-VIVO ASSESSMENT
}

\section{DALIA A. ELATY MOSTAFA ${ }^{1}$, MAHA K. A. KHALIFA ${ }^{2}$, SAMEH S. GAD ${ }^{3}$}

${ }^{1}$ Lecturer of Pharmaceutics, Faculty of Pharmacy, Pharmaceutics Department, October University for Modern Sciences and Arts (MSA) University, ${ }^{2}$ Lecturer of Pharmaceutics, Faculty of Pharmacy, Girls Branch, Al-Azhar University, Cairo, Egypt, ${ }^{3}$ Lecturer of Pharmacology and Toxicology, Faculty of Pharmacy, Pharmacology and Toxicology Department, October University for Modern Sciences and Arts (MSA) University Email: mahakhalifa.pharmg@azhar.edu.eg

Received: 09 Jan 2020, Revised and Accepted: 17 Feb 2020

\section{ABSTRACT}

Objective: Zolmitriptan, a class of antidepressant drugs with poor bioavailability due to its first-pass metabolism. The aim of this study was to improve systemic bioavailability and explore the brain targeting impact of nasal Zolmitriptan (Zol) solid lipid nanoparticles (SLNs) gel for migraine treatment.

Methods: Stearic acid and cholesterol used as solid lipid and lecithin as a surfactant, emulsion solvent evaporation technique was used to produce Zolmitriptan SLNs. (Zol) SLNs were characterized for particle size, percent entrapment efficiency and in vitro drug release. Formula S6 showed greater percent entrapment efficiency (PEE), adequate particle size and sustained drug release behavior. Formula S6 was integrated into HPMC gel (3\%) to prepare nasal gel. Zol SLN nasal gel was subjected to histopathological study to ensure brain targeting.

Results: It was observed that all prepared Zol SLNs were in the nano-sized range with a polydispersity index of $<0.5$. In the cholesterol/lecithin combination, higher PEE\%, better stability, and less agglomeration inclination were discovered. Results of the release profiles showed that developed Zol-SLNs were able to release Zolmitriptan in a sustained manner. Histopathological study of the brain tissues showed that Zolmitriptan SLN nasal gel can reach brain cells and localized for $24 \mathrm{~h}$ although the hydrophobicity of the target drug.

Conclusion: Intranasal administration of Solid lipid nanostructure of Zolmitriptan through the olfactory pathway in which it travels from the nasal cavity to brain tissue achieved drug targeting potential of about $90 \%$ compared with conventional Zolmitriptan tablets. The small particle size helped them to squeeze themselves through the small opening in the olfactory neurons to the brain via different endo-cystic pathways of neuronal cells in nasal tissue membranes.

Keywords: Zolmitriptan, Solid lipid nanoparticles (SLNs), Migraine, Histopathological examination, Brain targeting

(C) 2020 The Authors. Published by Innovare Academic Sciences Pvt Ltd. This is an open-access article under the CC BY license (http://creativecommons.org/licenses/by/4.0/) DOI: http://dx.doi.org/10.22159/ijap.2020v12i2.36812. Journal homepage: https://innovareacademics.in/journals/index.php/ijap

\section{INTRODUCTION}

Migraine is a disabling neurovascular disease with mostly unilateral throbbing headaches with a host of neurological symptoms including hypersensitivity to light, sound, smell, nausea and variety of autonomic and cognitive, emotional and motor disturbances [1]. Migraine mechanism suggests that vasoactive peptides will be released early in the attack from the primary sensory nerve terminals that innervate meningeal blood vessels. These peptides activate perivascular trigeminal nerves and cause dilatation of arteries in the meninges as well as perivascular inflammation and extravasation of plasma proteins [2]. Several triptans are 5HT1B/1D receptor agonists commonly prescribed for migraine headaches. Zolmitriptan is $4(\mathrm{~S})-4$ [3-(2-dimethyl aminoethyl)-1H5-indolyl-methyl]-1,3-oxazolan 2 one, potent and selective serotonin (5-HT1B/1D) receptor agonist which causes vasoconstriction of the blood vessels of the brain [3]. Also, reducing sterile inflammation associated with zolmitriptan antidromic neuronal transmission is another mechanism by which acute migraine attacks have been reported to be relieved [4]. It is currently available as a conventional tablet, an orally disintegrating tablet and a nasal spray (2.5 mg and $5 \mathrm{mg}$ per dose) [5]. Zolmitriptan's absolute bioavailability is up to $40 \%$ for both oral and nasal dosage forms and has a very brief half-life of $1-2 \mathrm{~h}$ with first-pass metabolism and rapid hepatic and renal clearance. This makes the oral route unsatisfactory [6]. Zolmitriptan nasal spray administration resulted in a quicker onset of action. However, clinical evidence shows no significant improvement in other pharmacokinetic parameters, such as half-life, bioavailability and therapeutic gain, over the oral dosage forms [4]. For these reasons, our objective was to overcome the abovementioned drawbacks by formulating Zolmitriptan's nasal gel delivery system for brain targeting. The present research focuses on the design of Zolmitriptan solid lipid nanoparticles (SLNs) to be delivered to the brain via an intranasal route. Solid lipid nanoparticles provide an enhancement of nose-to-brain drug delivery as they enable hydrophilic molecules to pass through the lipophilic BBB. They can also safeguard the encapsulated drug against biological and/or chemical degradation [7]. The delivery of drugs to the brain is a major challenge owing to the presence of the blood-brain barrier (BBB) [8]. Various techniques have been used to enhance drug delivery to the brain. One of these techniques includes nanotechnology-based drug transport. The intranasal route used for the delivery of many drugs to the central nervous system (CNS) [9]. Intranasal delivery does not require any modification of drugs [10].

Solid Lipid Nanocarriers (SLNs) enhance the permeability of drugs owing to lipid and surfactant content and thus improve bioavailability through oral, parenteral, dermal, nasal, ocular and pulmonary routes of administration due to their tiny size [11]. Their nano-size enables them to be transcellular transported to the brain via olfactory neurons through the different endocytic pathways of neuronal cells in the olfactory membrane [12]. We aimed to improve the systemic bioavailability of Zol due to the first-pass metabolism and explore the brain targeting the impact of nasal Zol (SLNs) gel for migraine treatment.

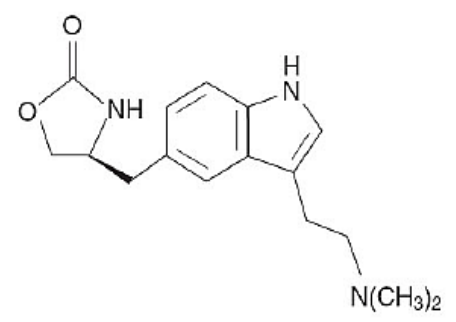

Scheme 1: Zolmitriptan structure 


\section{MATERIALS AND METHODS}

\section{Materials}

Zolmitriptan, HPMC E15 and Trimethylamine were kindly given as a blessing from Pharmaceutical Industries, EIPICO (Cairo, Egypt). Dimethylsulfoxide (DMSO), Stearic acid, Cholesterol, and Lecithin were acquired from Sigma Aldrich (St. Louis, USA).

\section{Methods}

\section{Formulation of SLNs of zolmitriptan}

Formulations investigated in the present work are listed in [table 1]. The blank-and-drug loaded SLNs were prepared by the emulsion solvent evaporation method (o/w) followed by homogenization [1315]. Stearic acid or Soy lecithin and cholesterol mixture were accurately weighed and dissolved in $10 \mathrm{ml}$ DMSO as the internal oil phase. Then heated above the melting point of lipid at $70{ }^{\circ} \mathrm{C}$. An aqueous surfactant solution of zolmitriptan was prepared by dissolving zolmitriptan in $5 \mathrm{ml}$ methanol and adding it with tween 80 to $20 \mathrm{ml}$ water. The organic phase was then poured drop by drop into a homogenizer tube containing $20 \mathrm{ml}$ of the hot aqueous phase. The mixture was then homogenized (Remi Instruments Pvt. Ltd, Mumbai, India) for $30 \mathrm{~min}$ at $3000 \mathrm{rpm}$ to form a primary emulsion $(\mathrm{o} / \mathrm{w})$ and stirred to extract the organic solvent into the continuous phase and for proper solidification of SLNs. The stirring was continued (2-3 h) at $3000 \mathrm{rpm}$ to get SLN dispersion.

\section{The standard calibration curve and specificity of the analytical} method used for Zolmitriptan determination

Zolmitriptan percentage entrapped in SLNs and the amount released was measured utilizing a UV spectroscopy (Jenway spectrophotometer, Model 6105UV/Vis, England). The maximum absorption of Zolmitriptan in phosphate buffer $\mathrm{pH} 6.8$ was found to be at $283 \mathrm{~nm}[16,17]$. Zolmitriptan calibration curve in $\mathrm{pH} 6.8$ phosphate buffer at a range of 1$10 \mathrm{ug} / \mathrm{ml}$ at $283 \mathrm{~nm}$ was plotted. The specificity of the analytical method was accomplished using a blank formula (Blank SLNs) prepared from all the excipients used except drug to verify whether any element of the formulation or dissolution medium could interfere with the absorption of Zolmitriptan at a chosen wavelength.

\section{Percent entrapment efficiency (PEE) of zolmitriptan in SLNs}

A volume of $2 \mathrm{ml}$ of each Zolmitriptan loaded SLNs was centrifuged at $15000 \mathrm{rpm}$ for $45 \mathrm{~min}$ to separate the lipid and aqueous phase. The supernatant was then diluted with methanol and filtered through $40 \mathrm{um}$ filter paper. The amount of unentrapped Zolmitriptan in the supernatant was determined spectrophotometrically at $\lambda_{\max } 283$ [18]. The EE percentage was calculated using the following equation where $\mathrm{Wi}$ is the amount of initial drug and Wf is the amount of free drug: $[19,20]$. PEE values given are the averages of three estimations.

$$
\text { EE\% }=\frac{\text { Wi-Wf }}{\text { Wi }} \times 100
$$

Particle size, polydispersity index (PDI) and zeta potential analysis

Particle size estimation of the dispersions performed utilizing a Zeta-sizer 3000 PCS (Malvern Instr., England) outfitted with a $5 \mathrm{~mW}$ helium-neon optical device. Estimations were made at $25^{\circ} \mathrm{C}$, edge 90 ${ }^{\circ}$, run time in any event $180 \mathrm{sec}$. The samples were appropriately dispersed in deionized water preceding the estimations. The particle size values given are the averages of 3 estimations over 5 min each.

\section{Atomic force microscopy (AFM)}

It is an ultra-high-resolution scanning probe microscopy (SPM). The morphology of SLNs was examined by (AFM) (Nanosurf Easyscan 2 AFM). The experiments were conducted in the water at room temperature $\left(20 \pm 1^{\circ} \mathrm{C}\right)$ under atmospheric pressure operating in non-contact mode. For this analysis, triangular silicon tips have been used. This cantilever's resonant frequency was about $120 \mathrm{kHz}$.

\section{Transmission electron microscopy (TEM)}

TEM (Philips, Tecrai10, Dutch) was used to examine the shape and surface morphology of the prepared zolmitriptan SLNs using a 300mesh carbon-coated copper grid and phosphotungstic acid (1\%; $\mathrm{w} / \mathrm{v}$ ) as a negative stain. After being stained, samples were allowed to dry at room temperature for $10 \mathrm{~min}$ for investigation.

\section{Scanning electron microscopy (SEM) studies}

Scanning electron microscopy (SEM) was used to study the morphological characteristics and texture of SLNs by JSM5910 (JEOL, Japan). SEM micrographs were recorded at a magnification of $60,000 \mathrm{x}$ and an accelerating voltage of $20 \mathrm{kV}$.

\section{Fourier transfer infrared (FT-IR) spectroscopy studies}

Using FT-IR studies (IR Prestige 21 Shimadzu, Japan) the potential interaction between the solid lipid core and the incorporated drug was investigated. The FT-IR analysis of Zolmitriptan, physical mixtures and zol loaded SLNs in a ratio of 1:1), blank-SLNs were performed with a resolution of $2 \mathrm{~cm}^{-1}$ in the range between 4,000 and $500 \mathrm{~cm}^{-1}$.

\section{In vitro drug release of zolmitriptan from SLNs and kinetic analysis of the release data}

The in vitro drug release of Zolmitriptan from SLNs was performed utilizing the dialysis bag technique [21]. In $12 \mathrm{~h}$ distilled water presoaked cellulose dialysis bag (cellulose membrane, 12,000-14000 D cut off molecular weight, Visking ${ }^{\circledR}$, Medicell, London, UK), Zolmitriptan loaded SLNs dispersion equivalent to $10 \mathrm{mg}$ of Zolmitriptan were accurately weighed and placed inside the dialysis bag and both ends of the bag were strongly closed. The bag was immersed into a beaker containing $50 \mathrm{ml}$ phosphate buffer solution (PBS) of $\mathrm{pH} 6.8$ which served as the receptor cell and placed in a shaker water bath at $37 \pm 0.5^{\circ} \mathrm{C}$ and agitated at $100 \mathrm{rpm}$ [22]. At time intervals from 0.5 to $5 \mathrm{~h}, 3 \mathrm{ml}$ were removed from the receptor cell for each sample and replaced by equivalent volumes of the fresh release medium and maintained at a similar temperature. Tests were estimated spectrophotometrically at $\lambda_{\max } 283 \mathrm{~nm}$ against equivalent phosphate buffer as a blank using Jenway spectrophotometer (Model 6105UV/Vis, England) [23]. The quantities of the released drug were calculated on the idea of the previously made calibration curve. Triplicate samples were analyzed and the average concentration was adopted. In vitro drug release data of Zolmitriptan from prepared SLNs were fitted to various kinetic models and analyzed to explain the mechanism of drug release.

\section{Physical stability studies}

The selected Zolmitriptan loaded SLN formula (S6) was evaluated for stability by storing and sealing in well-closed containers at $5 \pm 1,25 \pm 1$ and $45 \pm 1{ }^{\circ} \mathrm{C}$ for three weeks. The stability study was performed according to different parameters including; physical appearance, \%EE, particle size (PS), and zeta potential (ZP). The changes in \%EE, particle size, and zeta potential against storage time were monitored.

\section{Formulation of zolmitriptan loaded SLNs gel}

Formulation of Zolmitriptan loaded SLN that gave maximum entrapment, small particle size, small PDI, high zeta potential and better-sustained release effect was selected for preparation nasal gel (Tables 2 and 3). HPMC E15 polymer was used as a gelling agent for SLNs dispersion. A 3\% concentration of HPMC E 15 polymer was used. HPMC E15 was added to the nanoparticle dispersion under overhead stirring at $250 \mathrm{rpm}$ then, the dispersion was neutralized using $0.05 \%$ $(\mathrm{w} / \mathrm{w})$ triethanolamine. The gel was allowed to stand overnight to remove the entrapped air. The same gel formula was used to prepare a control gel containing pure drug at the same concentration.

\section{Characterization of Zol SLNs nasal gel}

Visual appearance, $\mathrm{pH}$ and drug content of zolmitriptan loaded SLNs nasal gel

Visual appearance was observed for the detection of any particular matter. The $\mathrm{pH}$ was measured using a calibrated digital $\mathrm{pH}$ meter (Model 420, ORION, USA) utilizing a $\mathrm{pH}$ of 4.0 and 7.0 standard buffers before use. The $\mathrm{pH}$ was noted by bringing the electrode close to the surface of the formulations and allowing it to equilibrate for one minute. The $\mathrm{pH}$ of the gel was measured at $25^{\circ} \mathrm{C}$. Gel was then diluted with methanol and filtered through 40 um filter paper. The 
amount of Zolmitriptan was determined spectrophotometrically at $\lambda$ $\max 283$ to determine drug content.

\section{Viscosity study of zolmitriptan loaded SLNs nasal gel}

Viscosity of the gel was measured on Brookfield viscometer (DVII+Pro Viscometer, Middleboro, USA). The gel formula was placed in a small sample adaptor, and the viscosity was measured using spindle LV-63 at $20 \mathrm{rpm}$.

\section{In vitro drug release study}

The dialysis bag diffusion was used to study Zolmitriptan loaded SLNs gel in vitro release. Zolmitriptan loaded SLNs gel (equivalent to $10 \mathrm{mg}$ of the drug) were put in the dialysis bag. The method was performed as shown before at time intervals from 1 to $24 \mathrm{~h}$, a placebo SLNS gel experiments were conducted. Samples were removed at predetermined time intervals from the receptor compartment and replaced by the fresh medium. The dissolved drug quantity was determined with UV spectrophotometry at $\lambda_{\max } 283$ $\mathrm{nm}$ against a blank. All experiments were performed in triplicate.

\section{Histopathological study of brain tissue}

In vivo histopathological study of brain tissues carried out of the previously chosen S6 zolmitipitan SLNs gel and plain gel $3 \%$ of HPMC E 15 gel without drug) on male albino rats (adult/weighing $200-250 \mathrm{~g}$ ). The protocol of the study was approved by the October University of Modern Science and Arts (MSA) Ethics Committee (PT8/ec8/2018f). The animals were kept under standard laboratory conditions, received a balanced diet of commercially available pellet rat feed, water and libitum, i.e. temperature of $22 \pm 3$ _C and relative humidity of $30-70 \%$ under a 24 -hour light/dark cycle. The animals were housed in polypropylene cages, four animals per cage with free access to standard laboratory diet and water.

1-Group I: received plain gel (3\% of HPMC E 15 gel without drug) to serve as a negative control group and sacrificed after 1 hour.
2-Group II: received SLNs zolmitriptan gel for S6 and sacrificed after 1 hour

3-Group III: received plain gel (3\% of HPMC E 15 gel without drug) to serve as a negative control group and sacrificed after $4 \mathrm{~h}$

4-Group IV: received SLNs zolmitriptan gel for S6 and sacrificed after $4 \mathrm{~h}$.

5-Group V: received plain gel (3\% of HPMC E 15 gel without drug) to serve as a negative control group and sacrificed after $24 \mathrm{~h}$.

6-Group VI: received SLNs zolmitriptan gel for S6 and sacrificed after $24 \mathrm{~h}$.

Rats were sacrificed using thiopental $(50 \mathrm{mg} / \mathrm{kg})$ and brains were removed, cleaned with cold saline $(0.9 \%)$. Brain tissues fixed in $10 \%$ formalin and embedded in paraffin wax were sonicated at $6 \mu \mathrm{m}$ thickness on a rotatory microtome, then stained with eosin and hematoxylin [24]. The stained sections were observed using the digital microscope and digital photomicrographs by using the attached camera.

\section{Statistical analysis}

Statistical analysis was performed using the GraphPad Prism version 8.1.2 software utilizing analysis of variance (ANOVA) or the paired ttest, where appropriate, and statistical significance was set at $P<0.05$.

\section{RESULTS AND DISCUSSION}

The standard calibration curve and specificity of the analytical method used for Zolmitriptan determination

Zolmitriptan exhibits its maximum absorption at $283 \mathrm{~nm}$ and obeyed Beer's law in the range of $1-10 \mu \mathrm{g} / \mathrm{ml}$. There was a good linear relationship between the Zolmitriptan concentration and its absorption $\left(R^{2}=0.996\right)$.

Table 1: Formulation of SLNs of zolmitriptan

\begin{tabular}{|c|c|c|c|c|c|c|}
\hline \multirow[t]{2}{*}{ Ingredients } & \multicolumn{6}{|c|}{ Quantities \% (W/W) } \\
\hline & S1 & S2 & S3 & S4 & S5 & S6 \\
\hline Zolmitriptan (mg) & 100 & 100 & 100 & 100 & 100 & 100 \\
\hline Stearic acid (mg) & 1000 & 1250 & 1500 & - & - & - \\
\hline DMSO (ml) & 10 & 10 & 10 & 10 & 10 & 10 \\
\hline Cholesterol (mg) & - & - & - & 125 & 200 & 250 \\
\hline Tween $80(\mathrm{ml})$ & 2 & 2 & 2 & 2 & 2 & 2 \\
\hline Lecithin & - & - & - & 25 & 50 & 75 \\
\hline Double distilled water (ml) & 30 & 30 & 30 & 30 & 30 & 30 \\
\hline
\end{tabular}

\section{Percent entrapment efficiency (PEE \%) of zolmitriptan in SLNs}

The formulations could be arranged in descending order according to PEE as follows: S1 $(40.87 \pm 7.25 \%)>\mathrm{S} 2 \quad(55.04 \pm 6.14 \%)>\mathrm{S} 3$ $(58.97 \pm 9.87 \%)>$ S $4(65.32 \pm 5.25 \%)>S 5(78 \pm 4.25 \%)>$ S $6(82.45 \pm 4.25$ $\%)$ as shown [table 2]. We noticed that PEE \% was increased by raising the lipid concentration (stearic acid and cholesterol). This may be because the quantity of lipids used in low concentration formulae is not adequate to entrap the drug, while higher concentration formulae have an adequate lipid to entrap the accessible drug and thus produce maximum PEE\%. Singh et al. noted that owing to more lipid accessibility, the rise in lipid quantity to the optimum level improves the PEE\% in order to entrap the drug [25] also, the lipid types highly affected the PEE\%. The mixture of cholesterol and lecithin showed significantly greater levels of PEE than stearic acid ( $S 4, S 5$ and $S 6$ ) at $\mathrm{p}<0.001$. It could be explained by the length of cholesterol is about $13.8 \mathrm{~A}^{\circ}$, which is much shorter than the length of stearic acid. A shorter length could generate more random molecular packing, allowing more space to drug accommodation [26]. The increase in drug entrapment efficiency might be also, due to lecithin effect: high Tc (phase transition temperature), decrease in membrane permeability, therefore, preventing drug leakage, hence the increase in Zolmitriptan content within the prepared SLN [27]. Vijayan et al. showed that the entrapment efficiency (\%) was directly proportional to lecithin concentration and obviated that low entrapment efficiency was due to low affinity between drugs at different solvent (organic and aqueous) [13]. Manjunath and his colleague revealed that the entrapment efficiency of the drug in the lipids depended upon the variable characters such as miscibility, the solubility of the drug in the lipid melt, physical and chemical structure of the lipid matrix and crystalline state of lipid components [28]. Varshosaz et al. showed that by increasing the amount of cholesterol in SLN, the PEE is much increased [29].

Table 2: PEE, Z-average, PDI and zeta potential of SLNs

\begin{tabular}{lllllll}
\hline Formulae & S1 & S2 & S3 & S4 & S5 & S6 \\
\hline Percent Entrapment efficiency (PEE) \pm SD & $40.87 \pm 7.25$ & $55.04 \pm 6.14$ & $58.97 \pm 9.87$ & $65.32 \pm 5.25$ & $78 \pm 4.25$ & $82.45 \pm 4.25$ \\
Z-average (nm) \pm SD & $144.9 \pm 40.3$ & $218.5 \pm 23.3$ & $205.8 \pm 16.9$ & $469.5 \pm 26.1$ & $581.2 \pm 29.6$ & $248.8 \pm 9.1$ \\
Polydispersity index (PDI) \pm SD & $0.596 \pm 0.05$ & $0.813 \pm 0.05$ & $0.347 \pm 0.03$ & $0.2 \pm 0.06$ & $0.631 \pm 0.02$ & $0.371 \pm 0.05$ \\
Zeta potential $\pm S D$ & $-12.1 \pm 1.02$ & $-12.6 \pm 1.56$ & $-13.4 \pm 0.97$ & $-20 \pm 1.47$ & $-15.7 \pm 1.03$ & $-14.2 \pm 1.56$ \\
\hline
\end{tabular}

mean $\pm S D, n=3$ 


\section{Particle size, polydispersity index (PDI) and zeta potential analysis}

Particle size measurement was done to ensure that particles of the Zol-SLNs in the nanometer range. We observed that all prepared SLNs were in the nano-sized range (average particle size values ranged from $144.9 \pm 40.3 \mathrm{~nm}$ to $581.2 \pm 29.6 \mathrm{~nm})$, with a polydispersity index of $<0.5$ as shown in [table 2]. The particle size increased significantly with the increase of lipid concentration (Stearic acid and cholesterol) at $\mathrm{p}<0.0001$. The results indicate that the amount of stearic acid has a positive influence on the mean size of the SLN. It is logical that increased particle size was observed with higher amounts of stearic acid. Lack of sufficient surfactant to cover the particle surface is the likely reason for increased particle size [30]. The particle size of SLNs prepared from the combination of cholesterol and lecithin was significantly greater than those prepared from stearic acid at $\mathrm{p}<0.0001$, but by increasing the concentration of lecithin to $75 \mathrm{mg}$ it showed a greater reduction in particle size of cholesterol SLNS to $248.8 \pm 9.1$ at $\mathrm{p}<0.0001$, which could be attributed to the excessive surfactant molecules present rapidly cover the new surfaces reducing the surface tension and thus facilitating the particle partition during emulsification[31]. As a result, an increase in the concentration of surfactant (Lecithin) results in a reduction in the SLN particle size. The interaction between cholesterol and surface-active agent might increase in surface curvature of oil droplets and leading to the small size of SLN after cooling down [32]. Jain et al. observed that lecithin addition prevents the agglomeration of particles [33]. Measuring zeta potential allows for predictions of colloidal dispersion storage stability [33]. Particle aggregation is less likely to occur for charged particles with high zeta potential due to electric repulsion. Compared to SLNs formulations, formulations containing cholesterol and lecithin combination showed a significant greater zeta potential values at $\mathrm{p}<0.01$ as shown in table 2 . Severino et al. showed that stearic acid nanoparticles were negatively charged, but the zeta potential was rather low [34].

\section{Atomic force microscopy (AFM)}

AFM images of Zolmitriptan loaded SLN showed that lipid composition did not influence nanoparticle morphology. Nanoparticles appeared in [fig. 1] to be round-shaped and SLN's mean particle diameter appeared to be smaller (around $150 \mathrm{~nm}$ ) compared to the particle size estimate. Additionally, no roughness is observed on the particle surface.
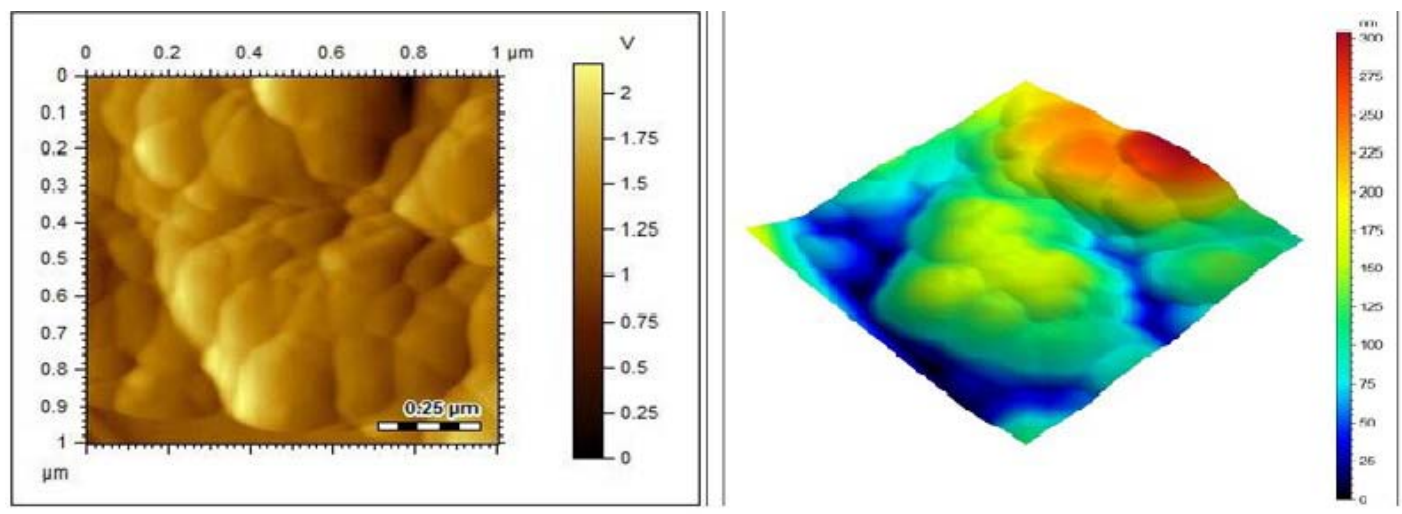

Fig. 1: AFM microphotographs of SLN

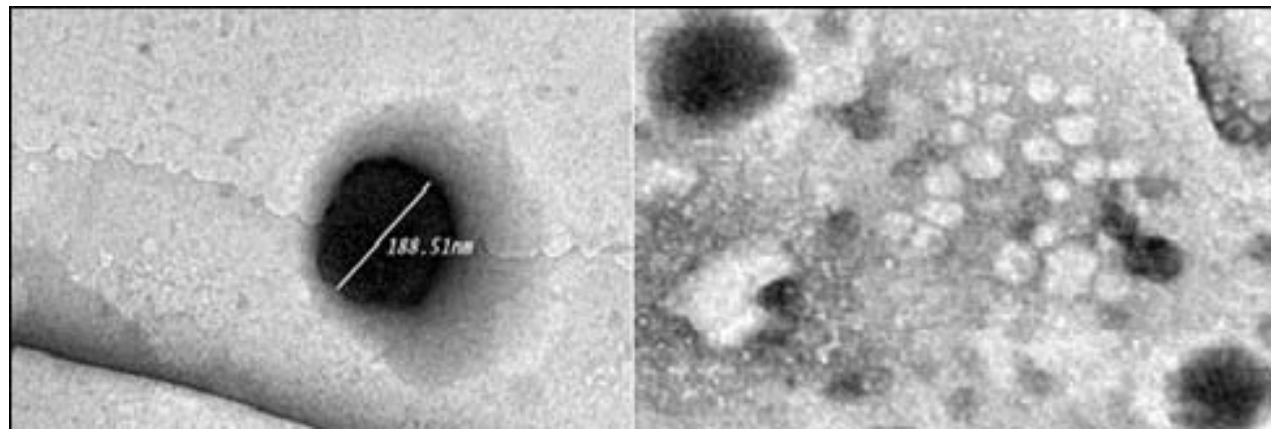

Fig. 2: Transmission electron micrograph of zolmitriptan loaded SLNs (S6)

\section{Transmission electron microscopy (TEM)}

TEM micrographs revealed the formation of nanoparticles of narrow particle size distribution [fig. 2]. Micrographs showed that the SLN were smooth, spherical and homogenous nanovesicles.

\section{Scanning electron microscopy (SEM) studies}

Fig. 3 showed a smooth and spherical shape of the particles. Also, particles generally agglomerated owing to carriers ' lipid nature, surfactant presence, and sample preparation before SEM analysis.

\section{Fourier transfer infrared (FT-IR) spectroscopy studies}

Zolmitriptan and lipid compatibility studies were carried out by FTIR, and the results were given in [fig. 4]. The pure zolmitriptan exhibits characteristic peaks at $3352 \mathrm{~cm}^{-1}$ corresponding to the aromatic secondary amine $\mathrm{N}-\mathrm{H} \quad$ stretching, $2974.23 \quad \mathrm{~cm}-1$ corresponding to aromatic C-H stretching, $1735.93 \quad \mathrm{~cm}-1$ corresponding to $\mathrm{C}=\mathrm{O}$ stretching and $1257.59 \mathrm{~cm}-1$ corresponding to C-N aliphatic amine stretching) as appeared in fig. 4. The FTIR spectra of Zolmitriptan and lipid physical mixture (cholesterol, Lecithin and stearic acid) exhibit the same characteristic peaks due the aromatic secondary amine $\mathrm{N}-\mathrm{H}$ stretching at $3348.42 \mathrm{~cm}-1, \mathrm{C}=0$ stretching at $1735.93 \mathrm{~cm}-1$, and $\mathrm{C}-\mathrm{N}$ aliphatic amine stretching at 1257.59, However they showed a slight shift in aromatic C-H stretching at 2931.8, 2978.09 and $2920.52 \mathrm{~cm}-1$ respectively. Thus, it is evident that all the characteristic peaks that were present in the spectra of pure drugs replicated almost in the same region in the spectra of Zolmitriptan and lipid physical mixture (cholesterol, Lecithin, and stearic acid) indicating that there is no significant interaction between the drugs and the lipids. 


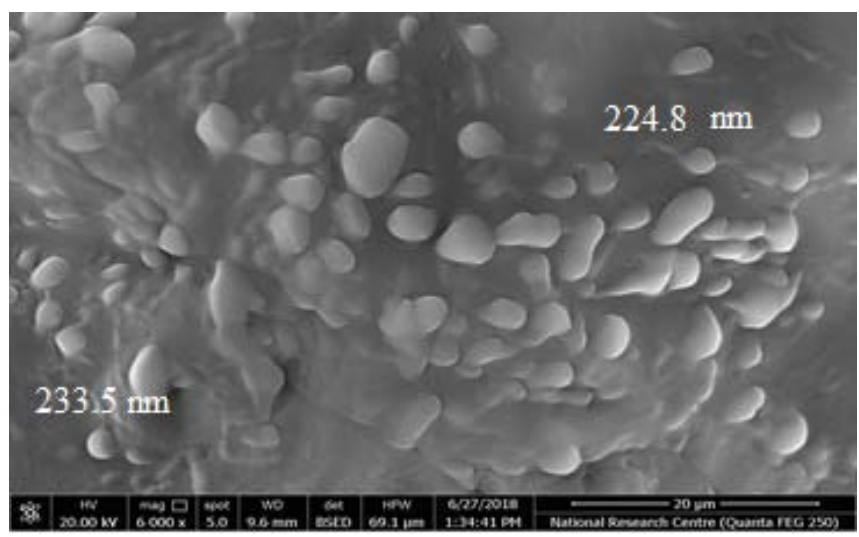

Fig. 3: SEM micrographs of Zolmitriptan loaded SLNs

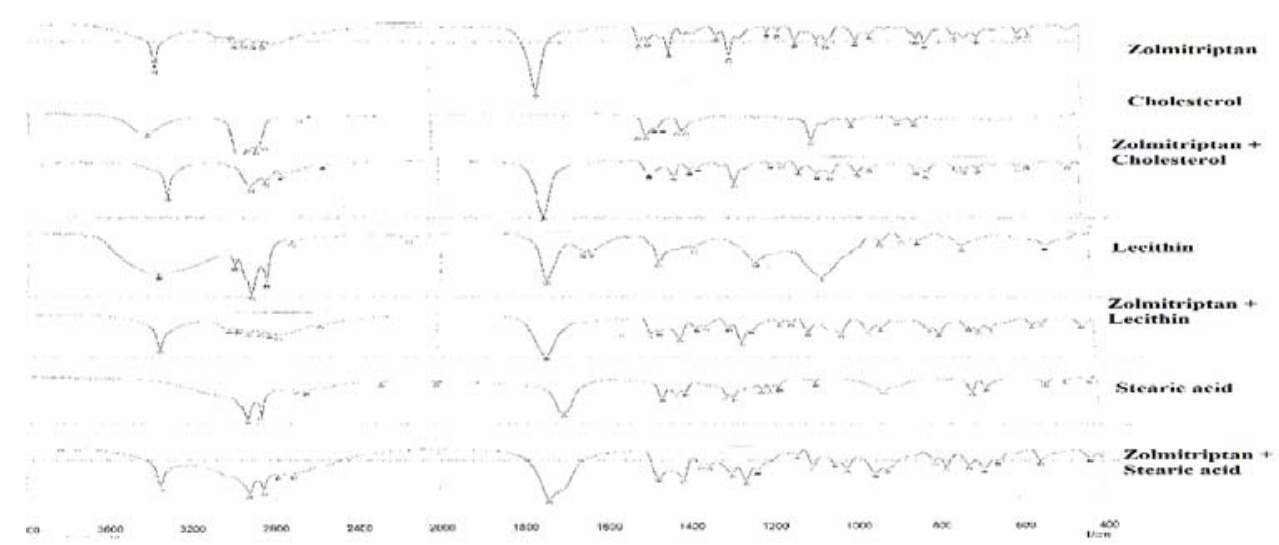

Fig. 4: FTIR spectrum of zolmitriptan, lipids, and their physical mixture

\section{In vitro release of zolmitriptan from SLNs and kinetic analysis of the release data}

In vitro drug release from the Zolmitriptan loaded SLNs was performed in PBS ( $\mathrm{pH} 6.8$ at $37^{\circ} \mathrm{C}$ ) using the dialysis bag technique over $5 \mathrm{~h}$ and the cumulative release percentages are illustrated in [fig. 5]. The release profiles of the investigated SLN formulations shows a biphasic behavior, an initial fast release that lasted for $0-1 \mathrm{~h}$, followed by a sustained slow release behavior. Burst release can be useful to improve the penetration of the drug, while, sustainedrelease supplied the drug over a prolonged period [33].

Cholesterol SLNs exhibited significantly higher initial burst release than Stearic acid at $\mathrm{p}<0.01$ and both followed by sustained release. The initial burst effect was ranged from $(22.01 \pm 1.23$ to $41.34 \pm 1.56)$ within one hour. The initial in vitro burst release was probably caused by the drug adsorbed on the nanoparticle surface or precipitated from the superficial lipid matrix [35]. Muntimadugu E et al. reported that initial burst release was mainly due to weakly bound drug on the surface of nanoparticles [36]. Baig et al. reported that the initial burst release rate was affected by the change of concentration of the lipid (Stearic acid and cholesterol) and surfactant (Lecithin) in the external phase. When the lipid concentration increased, the initial burst release rate decreased. Whereas surfactant concentration increases, the initial burst release rate increases due to the increased solubility of the drug in the external phase [37]. SLNs showed prolonged cumulative drug release percent and it decreased with increasing lipid concentration as shown in fig. 5. Due to the high concentration of drug in the inner phase [38]. The drug release of Zolmitriptan loaded SLNs was ranged from $50.24 \pm 5$.6to $75.75 \pm 5.6$ at $5 \mathrm{~h}$ SLNs suggests homogeneous entrapment of the drug throughout the systems. Furthermore, in vitro release profiles for the selected prepared SLNs were applied on various kinetic models (zero-order, first-order, and Higuchi equations), to fig. out the mechanism of drug release. The highest $r$ value for drug release was obtained for Higuchi's equation at $0.975,0.885,0.916,0.9655,0.976$ and 0.965 for S1, S2, S3, S4, S5 and S6 respectively. Release pattern of the drug from almost all SLNs formulations was best fitted into the Higuchi equation that describes the diffusion of the drug from homogenous and granular matrix systems [35]. Considering these observations, it was concluded that all the batches of SLNs had the potential for sustained drug delivery. This finding is in good agreement with the previous studies $[39,13]$.

Formulation of Zolmitriptan loaded SLNs that gave maximum entrapment efficiency percent, small particle size, small polydispersity index, high zeta potential, and better-sustained release effect were selected for physical stability studies, preparation of gel for nasal administration and brain histopathological studies. On that basis, we have chosen formula S6 for further investigations.

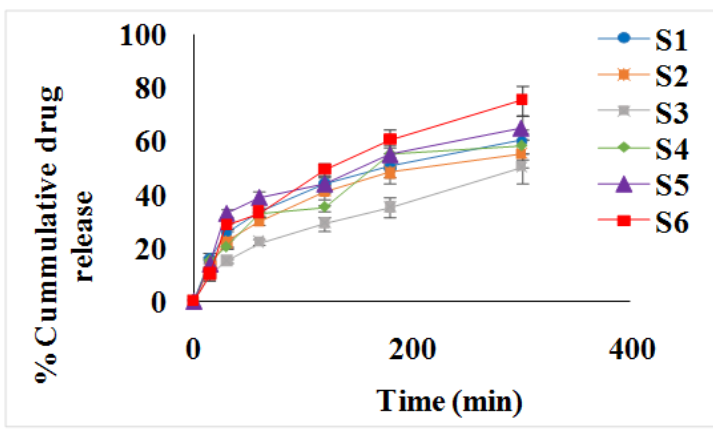

Fig. 5: In vitro drug release of zolmitriptan from SLNs; mean $\pm S D, n=3$ 


\section{Physical Stability studies}

During the storage period, there was no change in color or creaming and phase separation in the S6 formulation. There was a nonsignificant increase in particle size of SLN stored at $5{ }^{\circ} \mathrm{C}, 25^{\circ} \mathrm{C}$ and $45{ }^{\circ} \mathrm{C}$ after $3 \mathrm{w}$ at. At greater temperature $45{ }^{\circ} \mathrm{C}$, the entrapment efficiency percent was none significantly decreased compared to other storage circumstances. The non-significant change in size and $\% \mathrm{EE}$ due to the partial leakage of zolmitriptan from SLN. SLN stability also showed a non-significant decrease by raising the storage temperature as shown in the zeta potential outcomes. The results were presented in [table 3].

Table 3: Physical stability studies of SLNs

\begin{tabular}{lllll}
\hline Temperature & $\begin{array}{l}\text { Particle size (nm) } \\
\text { mean } \pm \text { SD }\end{array}$ & $\begin{array}{l}\text { PDI\% } \\
\text { mean } \pm \text { SD }\end{array}$ & $\begin{array}{l}\text { Entrapment efficiency \% } \\
\text { mean } \pm \text { SD }\end{array}$ & $\begin{array}{l}\text { Zeta potential (mv) } \\
\text { mean } \pm \text { SD }\end{array}$ \\
\hline $5 \mathrm{C}^{\circ}$ & $240 \pm 10.26$ & $0.43 \pm 0.88$ & $83 \pm 2.56$ & $-19.7 \pm 2.7$ \\
$25 \mathrm{C}^{\circ}$ & $254 \pm 14.26$ & $0.42 \pm 0.76$ & $79 \pm 1.94$ \\
$45 \mathrm{C}^{\circ}$ & $249 \pm 22.77$ & $0.41 \pm 0.56$ & $76 \pm 1.34$ & $-16 \pm 1.23$ \\
\hline
\end{tabular}

mean \pm SD, $n=3$

\section{Characterization of SLN gel}

\section{Visual appearance, $\mathrm{pH}$ and drug content of zolmitriptan loaded} SLN gel

The prepared gel of selected Zolmitriptan loaded SLN (S6) was white, smooth, homogenous with semisolid consistency, and show no syneresis. The $\mathrm{pH}$ of the Zolmitriptan loaded SLN formula was found to be $6.84 \pm 0.18$ with drug content in range $9 \mathrm{mg} / 0.5 \mathrm{~g}$ of gel $(90 \%)$ as shown in [table 4$]$.

\section{Viscosity study of zolmitriptan loaded SLN nasal gel}

The viscosity of Zolmitriptan loaded SLN nasal gel (gelled with HPMC E15) was recorded to be $3000 \mathrm{cp}$ as shown in [table 4]. It has to be noted that the viscosity of the gels is due to the polymer (HPMC E15) used.

\section{In vitro release study}

The gel showed an initial burst release of drug during the first 1 hour as discussed before. Following that, the drug entrapped into the gel was released gradually as shown in [fig. 6]. About $73.21 \pm 1.44 \%, 75.32 \pm 2.54 \%$ were released after 12 and $24 \mathrm{~h}$ respectively. In vitro release profiles for Zolmitriptan loaded SLN nasal gel was applied on various kinetic models (zero-order, firstorder, and Higuchi equations), to fig. out the mechanism of drug release. The highest $r$ value for drug release was obtained for Higuchi's equation at 0.967 compared to that of zero-order at 0.838 and first order at 0.912 . The mechanism of drug release was found to be Higuchi model. We concluded that Zolmitriptan loaded SLN nasal gel has a sustained drug release effect. This finding is in good agreement with the previous studies $[39,13]$.

Table 4: Zolmitriptan loaded SLN nasal gel characterization

\begin{tabular}{ll}
\hline Parameter & Zolmitriptan loaded SLNs nasal gel \\
\hline Visual Examination & White color with Good appearance \\
PH & $6.84 \pm 0.18$ \\
Drug Content & $90 \% \pm 7.25$ \\
Viscosity(cp) & $3000 \mathrm{cp}$ \\
\hline
\end{tabular}

mean $\pm S D, n=3$

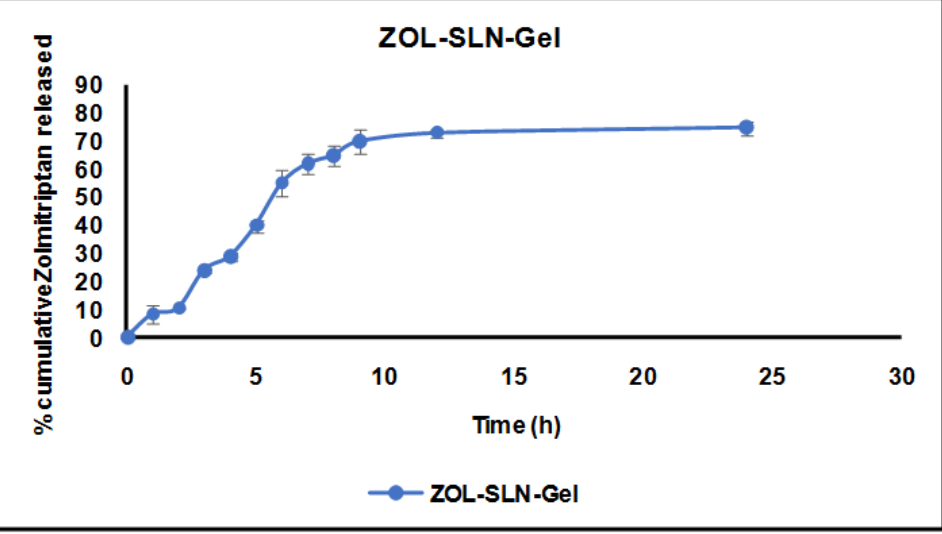

Fig. 6: In vitro drug release of zolmitriptan from SLNs nasal gel; mean \pm SD, $n=3$

\section{Histological examination of brain tissue}

Histopathological analysis was conducted on sections of brain tissue treated with formula S6 Zolmitriptan SLNs gel and control plain gel (3\% of HPMC E 15 gel) free from drug at a different time interval [fig. 7]. Histopathological examination of hippocampus normal pyramidal cells of brain tissue, (a) show no appearance of the drug after 1hour while, (b) showed a slight difference in cells condensation in CA2 layer after 1 hour. (C) Showed CA2 region with the intact pyramidal cell after four hours. While; (D) show a slight difference in cells structure in CA2 layer after four hours. (e) Showed normal neurons (arrowhead) and white matter (arrow) after $24 \mathrm{~h}$. (f) Showed spots of the drug appear around the cell after $24 \mathrm{~h}$. These promising results ensure that zolmitriptan drugs achieve higher brain targeting with $24 \mathrm{~h}$ which helps us to enhance efficacy in the treatment of migraine headaches specially that many patients suffer from nausea and/or vomiting that can make the oral treatment ineffective. 


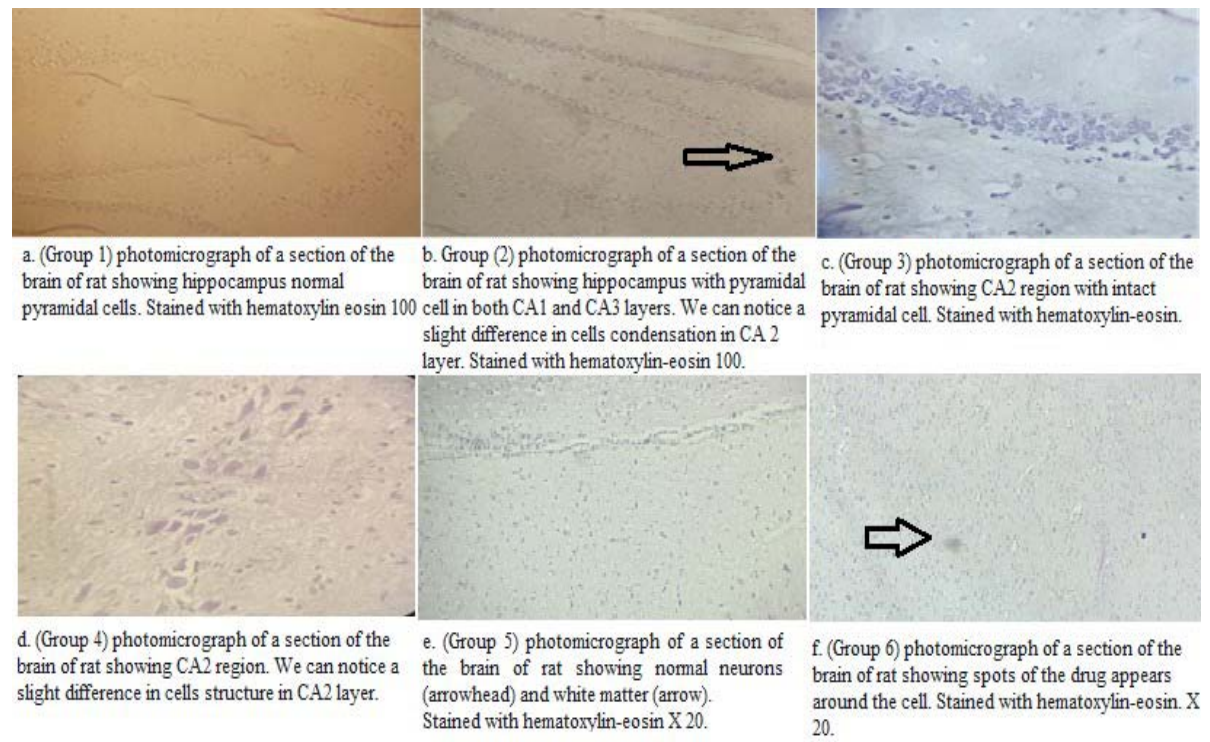

Fig. 7: Photomicrograph of a section of the brain of rat (a) received plain gel to serve as a negative control group and sacrificed after 1 hour. (b) Received SLNs zolmitriptan gel and sacrificed after $1 \mathrm{~h}$. (C) Received plain gel to serve as a negative control group and sacrificed after $4 \mathrm{~h}$. (D) Received SLNs zolmitriptan gel and sacrificed after $4 \mathrm{~h}$. (e) Received plain gel to serve as a negative control group and sacrificed after $24 \mathrm{~h}$. (f) Received SLNs zolmitriptan gel and sacrificed after $24 \mathrm{~h}$

\section{CONCLUSION}

Zolmitriptan (Zol) solid lipid nanoparticles (SLNs) gel success to reach the brain cells via permeation through nasal mucosa through the olfactory region thus; passing hepatic metabolism providing anti-migraine activity. The prepared Zol SLNs formulations were characterized for various parameters, and maximum entrapment efficiency was obtained with small particle size. Sustained release of ZMT SLNs was best fitted into the Higuchi equation that describes the diffusion of the drug from homogenous and granular matrix systems. Histological examination of brain tissue showed that Zolmitriptan achieved a higher brain targeting with $24 \mathrm{~h}$, which help us to enhance efficacy in the treatment of migraine headaches.

The brain targeting via nasal drug delivery system circumvent the pre-systemic metabolism thus increasing the bioavailability of zolmitriptan. Further, future clinical studies should be done on that technique to show the significance of an increase in bioavailability compared to commercial dosage forms.

\section{FUNDING}

Nil

\section{AUTHORS CONTRIBUTIONS}

All the authors have contributed equally.

\section{CONFLICT OF INTERESTS}

The authors report no conflicts of interest in this work.

\section{REFERENCES}

1. Youssef NA, Kassem AA, Farid RM, Ismail FA, EL-Massik MA, Boraie NA. A novel nasal almotriptan loaded solid lipid nanoparticles in mucoadhesive in situ gel formulation for brain targeting: preparation, characterization and in vivo evaluation. Int J Pharm 2018;548:609-24.

2. Wagh M, Bora SM. Development and evaluation of elastic niosomal formulation for migraine treatment using TRNA approach. Int J Pharm Sci Res 2018;9:4591-600.

3. Raza A, Ansari TM, Niazi SB. A novel spectrophotometric method for the determination of zolmitriptan in pharmaceutical formulations. I Chin Chem Soc 2007:54:1413-7.

4. Jain R, Nabar S, Dandekar P, Patravale V. Micellar nanocarriers: potential nose-to-brain delivery of zolmitriptan as novel migraine therapy. Pharm Res 2010;27:655-64.
5. Shiledar RR, Tagalpallewar AA, Kokare CR. Formulation and in vitro evaluation of xanthan gum-based bilayered mucoadhesive buccal patches of zolmitriptan. Carbohydr Polym 2014;101:1234-42.

6. Bayrak Z, Tas C, Tasdemir U, Erol H, Ozkan CK, Savaser A, et al. Formulation of zolmitriptan sublingual tablets prepared by direct compression with different polymers: in vitro and in vivo evaluation. Eur J Pharm Biopharm 2011;78:499-505.

7. Gupta S, Kesarla R, Chotai N, Misra A, Omri A. Systematic approach for the formulation and optimization of solid lipid nanoparticles of efavirenz by high-pressure homogenization using design of experiments for brain targeting and enhanced bioavailability. Biomed Res Int 2017. https://doi.org/10.1155/2017/5984014

8. WM Pardridge. The blood-brain barrier: bottleneck in brain drug development. NeuroRx 2005;2:3-14.

9. Singh GU, Rawat NI, Singh KI, Sarwal AM, Sinha VR Investigating the potential of an antidepressant intranasal mucoadhesive microemulsion. Int J Pharm Pharm Sci 2018;10:125-32.

10. Rodriguez A, Tatter SB, Debinski W. Neurosurgical techniques for disruption of the blood-brain barrier for glioblastoma treatment. Pharmaceutics 2015;7:175-87.

11. Begunm MY, Gudipati PR. Formulaton and evalution of dasatinib loaded solid lipid nanoparticles. Int J Pharm Pharm Sci 2018;10:15-20.

12. Jain K, Sood S, Gowthamarajan K. Optimization of artemetherloaded NLC for intranasal delivery using central composite design. Drug Delivery 2015;22:940-54.

13. Vijayan V, Aafreen S, Sakthivel S, Reddy KR. Formulation and characterization of solid lipid nanoparticles loaded Neem oil for topical treatment of acne. J Acute Disease 2013;2:282-6.

14. Garud A, Singh D, Garud N. Solid lipid nanoparticles (SLN): method, characterization and applications. Int Curr Pharm J 2012;1:384-93.

15. Mukherjee S, Ray S, Thakur RS. Solid lipid nanoparticles: a modern formulation approach in drug delivery system. Indian J Pharm Sci 2009;71:349.

16. Shelke S, Shahi S, Jalalpure S, Dhamecha D. Poloxamer 407 based intranasal thermoreversible gel of zolmitriptan-loaded nanoethosomes: formulation, optimization, evaluation and permeation studies. J Liposome Res 2016;26:313-23.

17. Panda N, Reddy AV, Reddy GS, Panda KC. Formulation design and in vitro evaluation of zolmitriptan immediate-release 
tablets using primojel and AC-Di-Sol. J Pharm Sci Res 2015;7:545.

18. Garud A, Singh D, Garud N. Design and optimization of solid lipid nanoparticles (SLNs) of zolmitriptan for the management of migraine. Ind J Pharm 2013;24:245-52.

19. Mostafa DA, Hashad AM, Abdelreheem AYM. Formulation and evaluation of novel brain targeting drug loaded in lipid-based nanoparticles through the intranasal route for Alzheimer. Int Res J Pharm 2019;10:21-7.

20. Balguri SP, Adelli GR, Majumdar S. Topical ophthalmic lipid nanoparticle formulations (SLN, NLC) of indomethacin for delivery to the posterior segment ocular tissues. Eur J Pharm Biopharm 2016;109:224-35.

21. Khalil RM, Abd-Elbary A, Kassem MA. Formulation and characterization of nystatin-loaded nanostructured lipid carriers for topical delivery against cutaneous candidiasis. BJPR 2013;4:490-512.

22. Fatouh AM, Elshafeey AH, Abdelbary A. Intranasal agomelatine solid lipid nanoparticles to enhance brain delivery: formulation, optimization and in vivo pharmacokinetics. Drug Des Dev Ther 2017;11:1815-25.

23. Abd-Elal RM, Shamma RN, Rashed HM, Bendas ER. Trans-nasal zolmitriptan novasomes: in vitro preparation, optimization and in vivo evaluation of brain targeting efficiency. Drug Delivery 2016;23:3374-86

24. Yassin GE, Amer RI, Fayez AM. Carpamazeine loaded vesicular structures for enhanced brain targeting via intranasal route: optimization, in vitro evaluation, and in vivo study. Int J Appl Pharm 2019;11:264-74.

25. Singh S, Dobhal AK, Jain A, Pandit JK, Chakraborty S. Formulation and evaluation of solid lipid nanoparticles of a water-soluble drug. Zidovudine Chem Pharm Bull 2010;58:650.

26. Kuo YC, Wang CC. Cationic solid lipid nanoparticles with primary and quaternary amines for release of saquinavir and biocompatibility with endothelia. Colloids Surfaces B: Biointerfaces 2013;101:101-5.

27. Abdelbary GA, Amin MM, Zakaria MY. Ocular ketoconazoleloaded proniosomal gels: formulation, ex vivo corneal permeation and in vivo studies. Drug Delivery 2017;24:309-19.

28. K Manjunath, JS Reddy, V Venkateswarlu. Solid lipid nanoparticles as drug delivery systems. Methods Find Exp Clin Pharmacol 2005;27:127.
29. Varshosaz J, Ghaffari S, Khoshayand MR, Atyabi F, Azarmi S, Kobarfard F. Development and optimization of solid lipid nanoparticles of amikacin by central composite design. J Liposome Res 2010;20:97-104.

30. Shah R, Eldridge D, Palombo E, Harding I. Optimisation and stability assessment of solid lipid nanoparticles using particle size and zeta potential. J Phys Sci 2014;25:59-75.

31. Ruckmani K, Sivakumar M, Ganeshkumar PA. Methotrexate loaded solid lipid nanoparticles (SLN) for effective treatment of carcinoma. J Nanosci Nanotech 2006;6:2991-5.

32. Asasutjarit R, Lorenzen SI, Sirivichayakul S, Ruxrungtham K, Ruktanonchai U, Ritthidej GC. Effect of solid lipid nanoparticle formulation compositions on their size, zeta potential and potential for in vitro pHIS-HIV-hugag transfection. Pharm Res 2007;24:1098-107.

33. Jain SK, Chourasia MK, Masuriha R, Soni V, Jain A, Jain NK, et al. Solid lipid nanoparticles bearing flurbiprofen for transdermal delivery. Drug Delivery 2005;12:207-15.

34. Severino P, Andreani T, Macedo AS, Fangueiro JF, Santana MH, Silva AM, et al. Current state-of-art and new trends on lipid nanoparticles (SLN and NLC) for oral drug delivery. J Drug Delivery 2012. Doi:10.1155/2012/750891.

35. Vivek K, Reddy H, Murthy RS. Investigations of the effect of the lipid matrix on drug entrapment, in vitro release, and physical stability of olanzapine-loaded solid lipid nanoparticles. AAPS PharmSciTech 2007;8:16-24.

36. Muntimadugu E, Dhommati R, Jain A, Challa VG, Shaheen M, Khan W. Intranasal delivery of nanoparticle encapsulated tarenflurbil: A potential brain targeting strategy for Alzheimer's disease. Eur J Pharm Sci 2016;92:224-34.

37. Baig MS, Ahad A, Aslam M, Imam SS, Aqil M, Ali A. Application of box-behnken design for preparation of levofloxacin-loaded stearic acid solid lipid nanoparticles for ocular delivery: optimization, in vitro release, ocular tolerance, and antibacterial activity. Int J Biol Macromol 2016;85:258-70.

38. Muller RH, MaEder K, Gohla S. Solid lipid nanoparticles (SLN) for controlled drug delivery-a review of the state of the art. Eur J Pharm Biopharm 2000;50:161-77.

39. El-Housiny S, Shams Eldeen MA, El-Attar YA, Salem HA, Attia D, Bendas ER, et al. Fluconazole-loaded solid lipid nanoparticles topical gel for the treatment of pityriasis versicolor: formulation and clinical study. Drug Delivery 2018;25:78-90. 